\title{
High levels of EphA3 expression are associated with high invasive capacity and poor overall survival in hepatocellular carcinoma
}

\author{
CHENG-YI LU ${ }^{1 *}$, ZHAO-XU YANG ${ }^{2 *}$, LIANG ZHOU ${ }^{3 *}$, ZHI-ZHONG HUANG $^{1}$, \\ HONG-TAO ZHANG ${ }^{2}$, JUN LI ${ }^{2}$, KAI-SHAN TAO ${ }^{2}$ and BAI-ZHI XIE ${ }^{4}$ \\ ${ }^{1}$ Department of Information, Tangdu Hospital, The Fourth Military Medical University, Xi'an, Shannxi 710032; \\ ${ }^{2}$ Department of Hepatobiliary Surgery, Xijing Hospital, The Fourth Military Medical University, \\ Xi'an, Shannxi 710032, ${ }^{3}$ Department of General Surgery, The 155 Central Hospital of PLA, Kaifeng, \\ He'nan 471000; ${ }^{4}$ The Fourth Military Medical University, Xi'an, Shannxi 710032, P.R. China
}

Received July 9, 2013; Accepted August 8, 2013

DOI: $10.3892 /$ or.2013.2679

\begin{abstract}
Although EphA3 expression has been associated with progression or prognosis in several types of tumors, the role of EphA3 in hepatocellular carcinoma (HCC) remains unknown. This study sought to investigate the clinicopathological and prognostic relevance of EphA3 expression in HCC as well as the underlying mechanisms responsible. EphA3 protein was mainly localized within the cytoplasm and at the cell membrane. High EphA3 expression was correlated with tumor size, tumor grade, metastasis, venous invasion and AJCC TNM stage $(\mathrm{P}<0.05)$, and patients with high levels of EphA3 expression were at a significantly increased risk for shortened survival time $(\mathrm{P}<0.05)$. In vitro, the downregulation of EphA3 expression decreased the invasive capacity of HCC cells via the regulation of VEGF. EphA3 may represent a novel candidate marker for patient prognosis as well a molecular target for HCC therapy.
\end{abstract}

\section{Introduction}

Although the mortality rate for hepatocellular carcinoma (HCC) has decreased, it is still one of the most common forms of cancer in China. Even with the advancement in diagnostic tools, surgical techniques and chemotherapy, a complete cure for HCC has not yet been achieved. Therefore, new diagnostic

Correspondence to: Professor Bai-Zhi Xie, The Fourth Military Medical University, Xi'an, Shannxi, 710032, P.R. China

E-mail: baizhixie@126.com

Professor Kai-Shan Tao, Department of Hepatobiliary Surgery, Xijing Hospital, The Fourth Military Medical University, Xi'an, Shannxi 710032, P.R. China

E-mail: baizhixie@126.com

*Contributed equally

Key words: hepatocellular carcinoma, EphA3, invasion, overall survival, clinicopathological correlation tools, novel therapeutic methods and new molecular markers of prognosis concerning this disease are urgently needed.

During cancer cell development, genes that encode oncoproteins, tumor-suppressors, and their regulators can be stochastically acquired and selectively accumulate mutations. Receptor tyrosine kinases (RTKs) are important regulators of signal transduction pathways and promote cell growth, survival, invasion and motility in tumors (1). Dysregulation of RTKs through mutation, amplification or overexpression, can increase kinase activity and ultimately oncogenic transformation. The generality of this paradigm of gain-of-function RTK signaling in cancer has been recently challenged by the discovery of the dual roles of Eph receptors in both promoting and inhibiting oncogenesis and tumor progression. Eph receptors constitute the largest subfamily of RTKs by far (2-4). A strikingly complementary pattern of expression between Ephs and ephrins in apposed cellular compartments has been observed in developing rhombomeres (5), along neural crest migration pathways (6) and in tissues throughout the entire developing mouse embryo (7). In contrast to most other RTKs, activation of Eph receptors does not typically lead to a proliferative response in cells, but rather to changes in cell shape, movement (2) and cell adhesion $(8,9)$. Numerous studies have reported that various Eph receptors are abnormally expressed in cancer. It has been confirmed that EphA1 protein is significantly associated with depth of invasion of gastric cancer, and patients with EphA1 upregulation experience a shorter survival time (10). Other studies suggest that the expression of EphA2 is higher in gastric cancer than that in normal mucosa, and is positively correlated with tumor TNM stage $(11,12)$. Overexpression of EphA7 has been observed more often in advanced gastric cancer, and EphA7 may have roles in the pathogenesis and development of gastric cancer (13). Several studies have shown that EphA3 expression is aberrantly regulated in hepatic cancer, lung cancer, renal cancer, colorectal cancer, melanoma and sarcoma (14-17). However, the relationship between EphA3 expression and survival in HCC patients has not been explored.

The present study used immunohistochemistry to investigate EphA3 protein expression and was the first to examine the potential relationship between EphA3 protein expression and 
patient prognosis in HCC. Furthermore, this study explored the role of EphA3 in the invasion and metastasis of HCC via its effect on the regulation of VEGF in vitro.

\section{Materials and methods}

Patients and tissue specimens. Tissue specimens from HCC and adjacent non-cancerous hepatic tissues (at least $1.5 \mathrm{~cm}$ away from the tumor) were collected from 101 patients who underwent surgical treatment for primary HCC at the Department of Hepatobiliary Surgery at Xijing Hospital (Xi'an, China) between 2003 and 2006. Specimens were obtained from patients who had not received preoperative treatments such as chemotherapy, ethanol injection, or transarterial chemoembolization. The study included 59 male and 42 female patients with a median age of 51.2 years (range, 32-71 years). The median size of the tumors was $6.3 \mathrm{~cm}$ (range, 2.7-13.1 cm). This study was approved by the Ethics Committee of the Fourth Military Medical University and conformed to the ethical guidelines of the 2004 Declaration of Helsinki. Written informed consent was obtained from each patient or from his/her legal guardians. Before the study was initiated, histopathological examinations were performed to confirm that there were enough cancer cells in the tumor samples and that no cancer cells had contaminated the non-cancerous hepatic tissues. All specimens were fixed in $10 \%$ formalin and embedded in paraffin, and $4-\mu \mathrm{m}$ serial sections were examined by immunohistochemistry. Clinical parameters such as gender, age, tumor location, tumor size, tumor grade, metastasis, satellite lesions, tumor number, AJCC TNM stage and $\alpha$-fetoprotein (AFP) were collected. The 38 cases diagnosed with metastasis included venous invasion $(n=26)$, bile duct tumor thrombi $(n=9)$ and lymph node metastasis $(n=3)$ and were verified by pathological analysis. The enrolled patients were followed up for 5 years to perform survival calculations.

Cell culture and reagents. The human liver non-tumor cell line (HL-7702) and the human HCC cell lines (HepG2, Huh-7, SMMC-7721 and MHCC97H) were cultivated in DMEM supplemented with $10 \%$ fetal calf serum (Sigma-Aldrich Chemical Co., St. Louis, MO, USA). The HCC cells were seeded into 6-well cell culture plates at a density of $1 \times 10^{5}$ cells/ well. Primary antibodies against EphA3, VEGF and GAPDH were purchased from Santa Cruz Biotechnology, Inc. (Santa Cruz, CA, USA). All secondary antibodies were obtained from Pierce Biotechnology, Inc. (Rockford, IL, USA). An SP immunostaining kit was purchased from Zymed (ZSGB; Beijing, China). EphA3 small interfering RNA (siRNA) and siRNA controls were obtained from Santa Cruz Biotechnology, Inc. Lipofectamine 2000 was purchased from Invitrogen Life Technologies (Carlsbad, CA, USA). All other chemicals and solutions were purchased from Sigma-Aldrich unless otherwise indicated.

Immunohistochemistry and evaluation of staining. Immunohistochemistry was performed using the avidin-biotinperoxidase method for all tissues. All sections were deparaffinized in xylene and dehydrated through a graded alcohol series prior to the blockade of endogenous peroxidase activity using $0.5 \% \mathrm{H}_{2} \mathrm{O}_{2}$ in methanol for $10 \mathrm{~min}$. Nonspecific binding was blocked by incubating the sections with $10 \%$ normal goat serum in PBS for $1 \mathrm{~h}$ at room temperature. Without washing, the sections were incubated with an anti-EphA3 antibody (1:50) in PBS at $4^{\circ} \mathrm{C}$ overnight in a humidified chamber. Biotinylated IgG (1:200; Sigma) was then added, and the sections were incubated for $2 \mathrm{~h}$ at room temperature. Detection was performed using a streptavidin-peroxidase complex. The brown color indicative of peroxidase activity was obtained by incubating with $0.1 \%$ 3,3-diaminobenzidine (Sigma) in PBS with $0.03 \% \mathrm{H}_{2} \mathrm{O}_{2}$ for $10 \mathrm{~min}$ at room temperature. The tissue specimens were scored independently by two pathologists, who were blinded to the clinicopathological results and patient outcome, using a previously described immunoreactivity scoring system (18). Based on the score, we divided all HCC specimens into 2 subgroups: the low expression group (score of 0-4) and the high expression group (score of 5-12).

Small interfering RNA transfection. According to the protocol supplied with the Lipofectamine 2000, HepG2 and MHCC97H cells were transfected with either EphA3 siRNA or control siRNA. siRNA-transfected cells were seeded into 6-well cell culture plates at a density of $1 \times 10^{5}$ cells/well. The cells were allowed to grow for an additional $24 \mathrm{~h}$ and were then harvested for further analysis.

Real-time reverse transcription-PCR. Total RNA was extracted and reverse-transcribed. The primers used for the PCR reaction were as follows: EphA3 forward primer (5'-CCA TGGACTGCCCAGCTGCC-3') and reverse primer (5'-CCT TGCGGCTGCACTGGTGA-3'); and GAPDH forward primer (5'-AAATCCCATCACCATCTTCC-3') and reverse primer (5'-TCACACCCATGACGAACA-3'). The primer sequences were verified by running a virtual PCR, and the primer concentrations were optimized to avoid primer-dimer formation. Additionally, dissociation curves were evaluated to avoid nonspecific amplification. Real-time PCR amplifications were performed using an Mx4000 Multiplex QPCR System (Stratagene, La Jolla, CA, USA) with 2X SYBR-Green PCR Master Mix (Applied Biosystems). Data were analyzed according to the comparative $\mathrm{C}_{\mathrm{t}}$ method and were normalized to GAPDH expression in each sample.

Protein extraction and western blotting. The cells were lysed in lysis buffer [50 mmol/1 Tris (pH 7.5), $100 \mathrm{mmol} / \mathrm{l} \mathrm{NaCl}$, $1 \mathrm{mmol} / \mathrm{l}$ EDTA, $0.5 \% \mathrm{NP} 40,0.5 \%$ Triton X-100, $2.5 \mathrm{mmol} / \mathrm{l}$ sodium orthovanadate, $10 \mu \mathrm{l} / \mathrm{ml}$ protease inhibitor cocktail, and $1 \mathrm{mmol} / \mathrm{l} \mathrm{PMSF}$ ] by incubating for $20 \mathrm{~min}$ at $4^{\circ} \mathrm{C}$. The protein concentration was determined using the Bio-Rad assay system (Bio-Rad, Hercules, CA, USA). Total proteins were fractionated using SDS-PAGE and transferred onto nitrocellulose membranes. The membranes were blocked with $5 \%$ nonfat dried milk or bovine serum albumin in 1X TBS buffer containing $0.1 \%$ Tween- 20 and then incubated with the appropriate primary antibodies. Horseradish peroxidase-conjugated anti-rabbit or anti-mouse $\mathrm{IgG}$ was used as the secondary antibody, and the protein bands were detected using the enhanced chemiluminescence detection system (Amersham Pharmacia Biotech). Quantification of the western blotting was performed using laser densitometry, and relative protein expression was then normalized to GAPDH levels. 

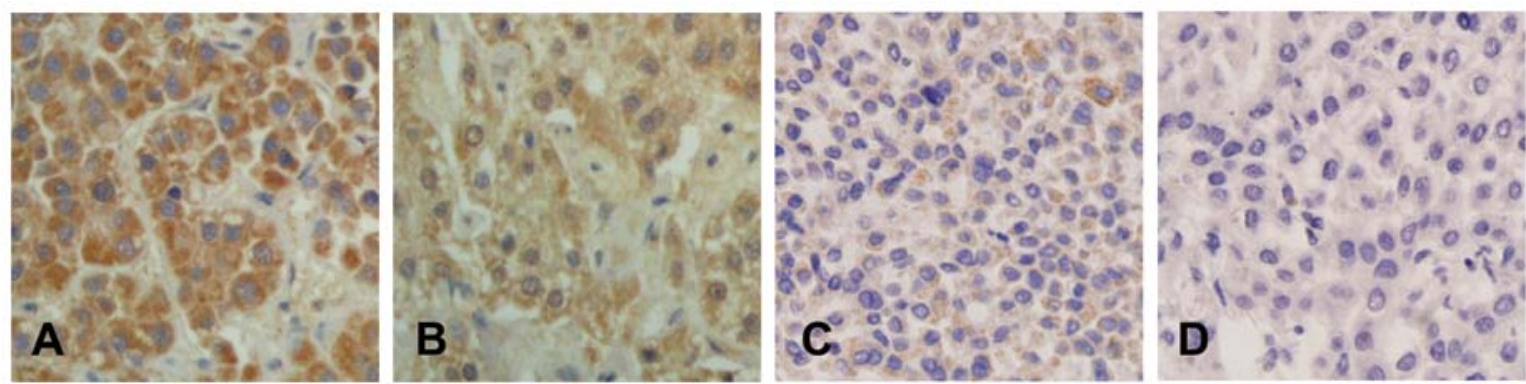

Figure 1. Different levels of EphA3 expression in HCC tissues. (A) Strong, (B) moderate, (C) weak and (D) negative. HCC, hepatocellular carcinoma.

Invasion assays. Cell invasion was analyzed using Matrigelcoated Transwell cell culture chambers $(8-\mu \mathrm{m}$ pore size $)$ (Millipore, Billerica, MA, USA). Briefly, the treated cells $\left(5 \times 10^{4}\right.$ cells/well) were serum-starved for $24 \mathrm{~h}$ and plated in the upper insert of a 24-well chamber in serum-free medium. Medium containing $10 \%$ serum as a chemoattractant was then added to the wells, and the cells were incubated for $24 \mathrm{~h}$. Cells on the upper side of the filters were mechanically removed using a cotton swab, after which the membrane was fixed with $4 \%$ formaldehyde for $10 \mathrm{~min}$ at room temperature, and stained with $0.5 \%$ crystal violet for $10 \mathrm{~min}$. Finally, invasive cells were counted at x200 magnification from 10 different fields in each filter.

ELISA assay. The enzyme-linked immunosorbent assay (ELISA) technique (Amersham, Buckinghamshire, UK) was used to quantify the activity of VEGF. The samples were thawed on ice, and all reagents were equilibrated to room temperature. All assays were carried out according to the manufacturer's instructions.

Statistical analysis. Statistical analysis was performed using SPSS 13.0 software (SPSS, Inc., Chicago, IL, USA). Each experiment was repeated at least 3 times, and all data were summarized and presented as the means \pm SDs. The differences between means were statistically analyzed using a t-test. The $\chi^{2}$ test for proportions was used to analyze the relationship between EphA3 expression and various clinicopathologic factors. Survival curves were calculated using the Kaplan-Meier method and compared using the log-rank test. Cox proportional hazard analysis was used for univariate and multivariate analysis to explore the effect of clinicopathological factors and the EphA3 expression on survival. P-values $<0.05$ were considered to indicate statistically significant results.

\section{Results}

EphA3 immunohistochemistry. EphA3 expression was mainly localized within the cytoplasm and at the cell membrane. No significant EphA3 expression was noted in the adjacent non-cancerous hepatic tissues, with only weak staining for EphA3 at the cell membrane and in the cytoplasm. As shown in Fig. 1, the expression of EphA3 differed between HCC tissues. EphA3 staining was negative in 19 samples of HCC, whereas weak positive staining was detected in 27 samples of $\mathrm{HCC}$, moderate positive staining was detected in 23 samples of

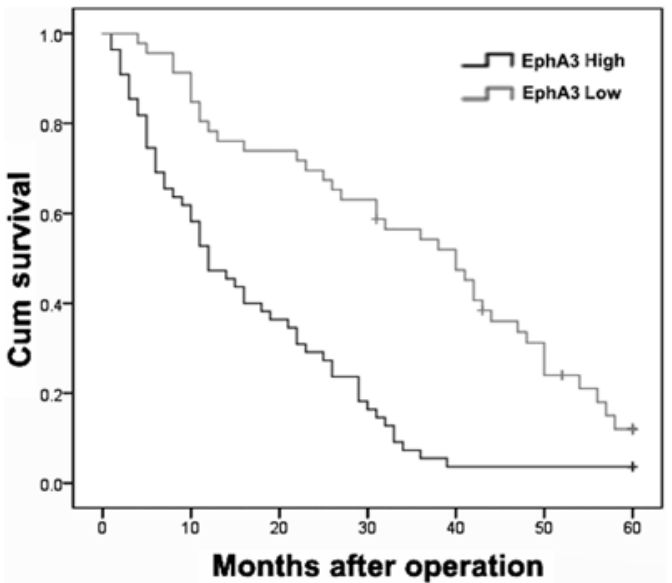

Figure 2. Kaplan-Meier statistical analysis of postoperative survival curves according to EphA3 expression.

HCC, and strong positive staining was detected in 32 samples of HCC.

Relationship between EphA3 expression and clinicopathological characteristics. The pathological factors examined for 101 cases of HCC included gender, age, tumor location, tumor size, tumor grade, metastasis, tumor number, AJCC TNM stage and AFP. In cases diagnosed with metastasis, we also analyzed vascular invasion. For this analysis, we divided the 101 patients into 2 subgroups: a high EphA3 expression group $(n=55)$ and a low EphA3 expression group $(n=46)$. The relationship between EphA3 expression and the clinicopathological factors is summarized in Table I. The results demonstrated that high EphA3 expression was strongly correlated with tumor size $(\mathrm{P}=0.006)$, tumor grade $(\mathrm{P}=0.003)$, metastasis $(\mathrm{P}=0.003)$, venous invasion $(\mathrm{P}=0.008)$ and AJCC TNM stage $(\mathrm{P}<0.001)$. However, there were no significant associations between EphA3 expression and the other pathological factors examined $(\mathrm{P}>0.05)$. These results indicate that EphA3 may be involved in the differentiation, invasion and metastasis of HCC.

Correlation between EphA3 expression and prognosis of HCC patients. Since the level of EphA3 expression was correlated with tumor size, tumor grade, metastasis, venous invasion and AJCC TNM stage, we further speculated that the level of EphA3 expression may affect the prognosis of HCC patients. Kaplan-Meier postoperative survival curves 
Table I. Association of EphA3 expression with clinicopathologic factors of the HCC patients.

\begin{tabular}{|c|c|c|c|c|c|}
\hline \multirow[b]{2}{*}{ Tumor characteristics } & \multirow[b]{2}{*}{$\mathrm{n}$} & \multicolumn{2}{|c|}{ EphA3 } & \multirow[b]{2}{*}{ P-value } & \multirow[b]{2}{*}{$\chi^{2}$} \\
\hline & & $\begin{array}{l}\text { High, n }(\%) \\
(5-12 \text { score })\end{array}$ & $\begin{array}{c}\text { Low, n (\%) } \\
(0-4 \text { score })\end{array}$ & & \\
\hline All cases & 101 & $55(54.5)$ & $46(45.5)$ & & \\
\hline \multicolumn{6}{|l|}{ Gender } \\
\hline Male & 59 & $34(57.6)$ & $25(42.4)$ & 0.448 & 0.575 \\
\hline Female & 42 & $21(50.0)$ & $21(50.0)$ & & \\
\hline \multicolumn{6}{|l|}{ Age (years) } \\
\hline$\leq 50$ & 50 & $30(60.0)$ & $20(40.0)$ & 0.268 & 1.227 \\
\hline$>50$ & 51 & $25(49.0)$ & $26(51.0)$ & & \\
\hline \multicolumn{6}{|l|}{ Tumor location } \\
\hline Left & 53 & $29(54.7)$ & $24(45.3)$ & 0.956 & 0.003 \\
\hline Right & 48 & $26(54.2)$ & $22(45.8)$ & & \\
\hline \multicolumn{6}{|l|}{ Tumor size (cm) } \\
\hline$\leq 5$ & 38 & $14(36.8)$ & $24(63.2)$ & 0.006 & 7.620 \\
\hline$>5$ & 63 & $41(65.1)$ & $22(34.9)$ & & \\
\hline \multicolumn{6}{|c|}{ Tumor grade (differentiation) } \\
\hline Well & 33 & $25(75.8)$ & $8(24.2)$ & 0.003 & 8.968 \\
\hline Moderately or poorly & 68 & $30(44.1)$ & 38 (55.9) & & \\
\hline \multicolumn{6}{|l|}{ Metastasis } \\
\hline Yes & 38 & $28(73.7)$ & $10(26.3)$ & 0.003 & 9.082 \\
\hline No & 63 & $27(42.9)$ & $36(57.1)$ & & \\
\hline \multicolumn{6}{|l|}{ Venous invasion } \\
\hline+ & 26 & $20(76.9)$ & $6(23.1)$ & 0.008 & 7.126 \\
\hline- & 75 & $35(46.7)$ & $40(53.3)$ & & \\
\hline \multicolumn{6}{|l|}{ Satellite lesions } \\
\hline+ & 29 & $18(62.1)$ & $11(37.9)$ & 0.330 & 0.951 \\
\hline- & 72 & $37(51.4)$ & $35(48.6)$ & & \\
\hline \multicolumn{6}{|l|}{ Tumor number } \\
\hline Solitary & 68 & $35(51.5)$ & $33(48.5)$ & 0.387 & 0.748 \\
\hline Multiple & 33 & $20(60.6)$ & $13(39.4)$ & & \\
\hline \multicolumn{6}{|l|}{ AJCC TNM stage } \\
\hline I and II & 19 & $1(5.3)$ & $18(74.7)$ & $<0.001$ & 22.834 \\
\hline III and IV & 82 & $54(65.9)$ & $28(34.1)$ & & \\
\hline \multicolumn{6}{|l|}{$\operatorname{AFP}(\mathrm{ng} / \mathrm{ml})$} \\
\hline$\leq 400$ & 71 & $18(60.0)$ & $1(40.0)$ & 0.467 & 0.529 \\
\hline$>400$ & 30 & $37(52.1)$ & $34(47.9)$ & & \\
\hline
\end{tabular}

HCC, hepatocellular carcinoma; AFP, $\alpha$-fetoprotein.

were used to evaluate the overall survival rates of patients with HCC in comparison to their levels of EphA3 expression. The log-rank test showed that survival time was significantly different between the low and high EphA3 expression groups $(\mathrm{P}<0.001)$. The low EphA3 expression group demonstrated increased survival, whereas the high EphA3 expression group demonstrated reduced survival (Fig. 2). The cumulative 5-year survival rate was $31.2 \%$ in the low EphA3 expression group, whereas this rate was only $13.6 \%$ in the high EphA3 expression group.
A univariate Cox regression analysis also found that tumor grade, metastasis, venous invasion, satellite lesions, AJCC TNM stage and EphA3 protein expression were significantly associated with overall survival (Table II). Furthermore, to evaluate the potential of high EphA3 expression to serve as an independent predictor for overall survival among HCC patients, multivariate Cox regression analyses were performed. The results indicated that only metastasis and EphA3 expression could predict overall survival among HCC patients (Table II). 
Table II. Univariate and multivariate analysis for overall survival of the HCC patients.

\begin{tabular}{lcc}
\hline Tumor characteristics & Relative risk $(95 \%$ CI) & P-value \\
\hline Univariate & & \\
Gender & $1.024(0.674-1.556)$ & 0.911 \\
Age (years) & $1.075(0.711-1.625)$ & 0.732 \\
Tumor location & $0.948(0.628-1.432)$ & 0.800 \\
Tumor size & $1.328(0.857-2.058)$ & 0.204 \\
Tumor grade & $0.578(0.370-0.902)$ & 0.016 \\
(differentiation) & & \\
Metastasis & $11.292(6.203-20.557)$ & $<0.001$ \\
Venous invasion & $10.904(5.925-20.066)$ & $<0.001$ \\
Satellite lesions & $2.080(1.306-3.314)$ & 0.002 \\
Tumor number & $1.357(0.881-2.090)$ & 0.166 \\
AJCC TNM stage & $3.694(2.022-6.748)$ & $<0.001$ \\
AFP (ng/ml) & $0.739(0.470-1.162)$ & 0.191 \\
EphA3 & $2.927(1.876-4.569)$ & $<0.001$ \\
Multivariate & & \\
Tumor grade & $1.194(0.725-1.965)$ & 0.486 \\
(differentiation) & & \\
Metastasis & $5.917(2.762-12.676)$ & $<0.001$ \\
Venous invasion & $2.516(1.240-5.108)$ & 0.11 \\
AJCC TNM stage & $1.961(0.981-3.920)$ & 0.057 \\
EphA3 & $1.960(1.181-3.252)$ & 0.009 \\
\hline
\end{tabular}

95\% CI, 95\% confidence interval; HCC, hepatocellular carcinoma; AFP, $\alpha$-fetoprotein.

Downregulated expression of EphA3 by siRNA reduces the invasiveness of HCC cells. Since high expression of EphA3 was strongly correlated with metastasis $(\mathrm{P}=0.003)$ and venous invasion $(\mathrm{P}=0.008)$, we next sought to determine whether EphA3 was involved in invasion and metastasis in HCC. We first examined the expression levels of EphA3 in different HCC cells with different invasive capabilities. As another study reported, the invasive capacity of HepG2 cells was the lowest, whereas the invasive capacity of MHCC $97 \mathrm{H}$ cells was the highest (19). RT-PCR and western blot analysis showed that the expression levels of EphA3 mRNA and protein exhibited similar increased tendencies related to the invasive capability (Fig. 3A and B) in HCC cells. In HCC cells, siRNA was used to effectively decrease the expression of EphA3 mRNA and protein (Fig. 4A-C). Using Transwell cell culture chambers, we measured the invasiveness of EphA3 siRNA-transfected cells. As illustrated in Fig. 4D, the number of EphA3 siRNA-transfected HepG2 cells that migrated through the Transwell was significantly less than the number of control siRNA-transfected cells that migrated. In addition, the use of MHCC97H cells showed similar results (Fig. 4D). Thus, these data indicated that the downregulation of EphA3 by siRNA reduced the invasive capacity of HCC cells.

Downregulation of EphA3 decreases the protein expression and proteolytic activity of VEGF. To determine the potential
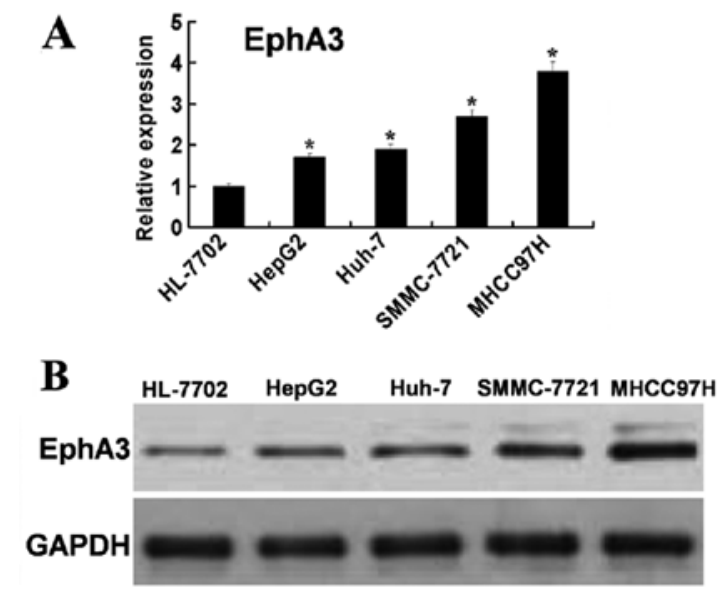

Figure 3. Expression of EphA3 in the liver non-tumor and HCC cells (A) RT-PCR analysis of the expression of EphA3 in liver non-tumor and HCC cells. (B) Western blot analysis of the expression of EphA3 in liver nontumor and HCC cells. The expression of EphA3 was normalized to GAPDH "P<0.05 compared with HL-7702 cells. HCC, hepatocellular carcinoma.

mechanism for the role of EphA3 in HCC cell invasion, we examined the effect of decreased EphA3 on VEGF. Using western blotting and ELISA, we found that the protein expression levels and proteolytic activity of VEGF were decreased in EphA3 siRNA-transfected HepG2 cells (Fig. 5A and B). In addition, the use of MHCC $97 \mathrm{H}$ cells showed similar results (Fig. 5A and C). These results indicated that EphA3 may participate in HCC cell invasion by regulating the expression and activity of VEGF. However, additional studies are needed to address why EphA3 can regulate protein expression levels and proteolytic activity of VEGF.

\section{Discussion}

Tyrosine kinases, which are the major regulators of signal transduction pathways, are associated with cellular proliferation, apoptosis and tumorigenesis (20). Eph receptor tyrosine kinases (Ephs) and their membrane-anchored ephrin ligands (ephrins) form a cell-cell system that is associated with cell-tocell adhesion or migration, angiogenesis and tumor vasculature in various human carcinomas $(21,22)$. During adulthood, many Ephs and their ligands (ephrins) are expressed in malignant tissues and are thought to participate in tumor invasion and metastasis $(23,24)$. EphA3 is a component of the Eph/ephrin tyrosine kinase system, which participates in blood vessel development (25) and play a potentially significant role in tumor angiogenesis (26). Changes in Eph A3 are likely to produce particular morphological and biological characteristics, such as cell growth and viability, loss of cell adhesion to fibronectin, cell migration and anti-apoptosis. Considerable evidence indicates that aberrant regulation of EphA3 and its genetic alterations are implicated in the development and progression of various types of cancers (27-30). Studies have shown that EphA3 expression is detected in B- and T-lymphoid tumor cell lines and in primary leukemias (31) and is associated with B- and T-cell malignancies (32-34). Furthermore, it has been reported that EphA3 is overexpressed in a range of tumors, such as lung cancer, renal cancer, colorectal cancer, melanoma 

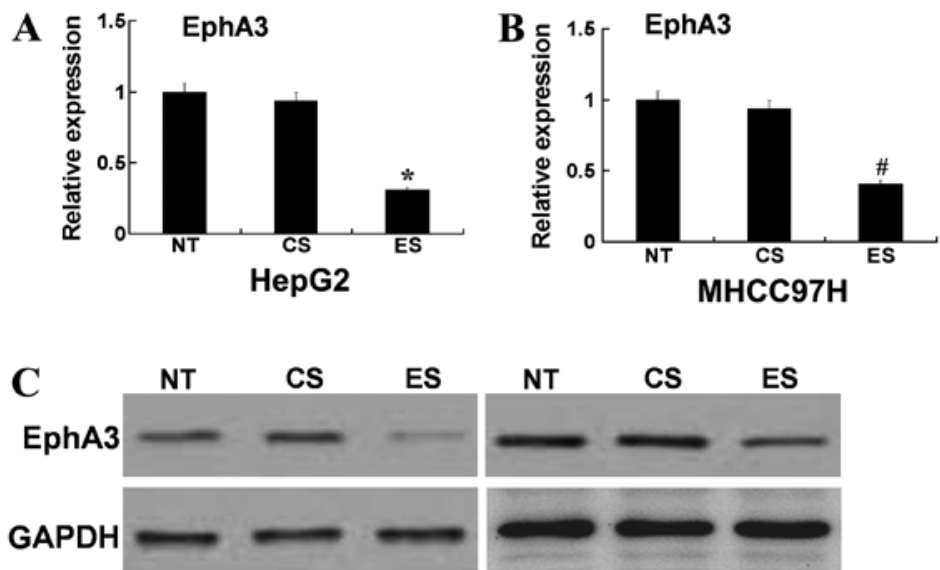

HepG2

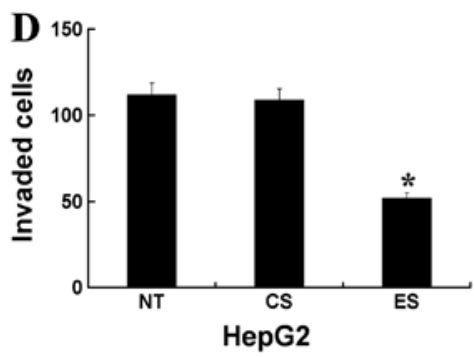

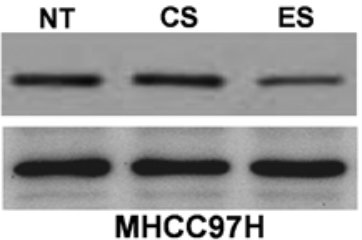

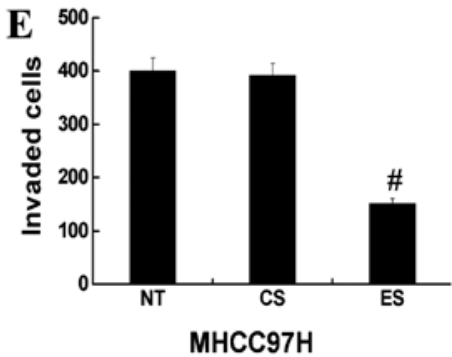

Figure 4. Inhibition of EphA3 by siRNA decreased the invasive capabilities of HCC cells in vitro. (A-C) RT-PCR and western blotting were performed to assess the expression of EphA3 in EphA3 siRNA-transfected HCC cell lines. The expression of EphA3 was normalized to that of GAPDH. (D and E) Inhibition of EphA3 decreased the invasive capabilities of HCC cells. ${ }^{*} \mathrm{P}<0.05$ compared to control siRNA-transfected HepG2 cells; ${ }^{*} \mathrm{P}<0.05$ compared to control siRNA-transfected MHCC97H cells. NT, non-transfection; ES, EphA3 siRNA-transfection; CS, control siRNA-transfection. HCC, hepatocellular carcinoma.

A

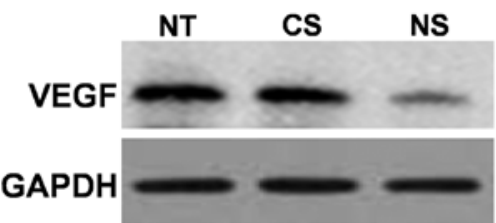

HepG2

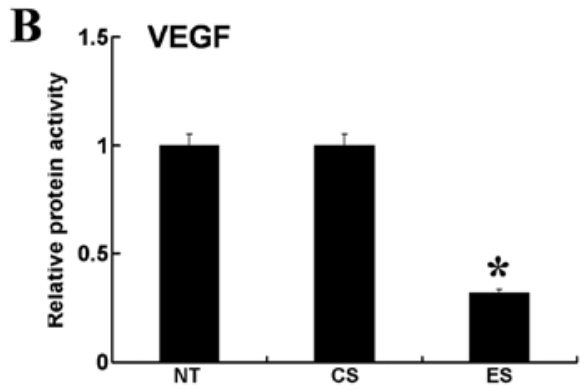

HepG2

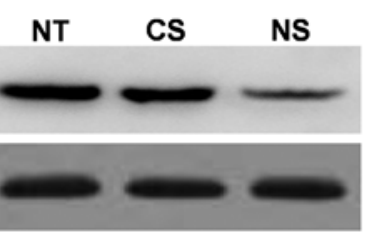

MHCC97H

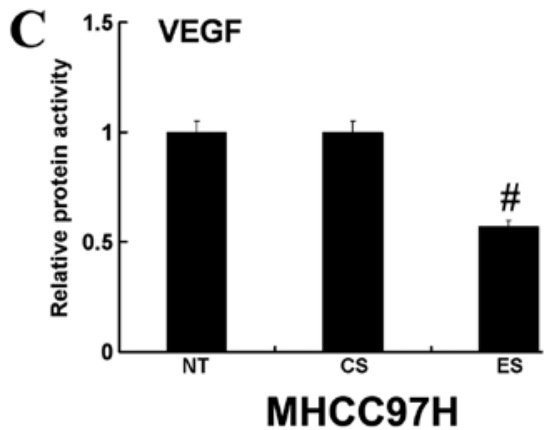

Figure 5. In HCC cells, the inhibition of EphA3 decreased the protein expression and proteolytic activity of VEGF. (A) The protein expression levels of VEGF were measured by western blotting. (B and C) The proteolytic activity of VEGF was measured by ELISA. The data represent the means \pm SD, ${ }^{*} \mathrm{P}<0.05$ compared to control siRNA-transfected HepG2 cells; ${ }^{\text {P }}<0.05$ compared to control siRNA-transfected MHCC97H cells. NT, non-transfection; ES, EphA3 siRNA-transfection; CS, control siRNA-transfection. HCC, hepatocellular carcinoma.

and sarcoma $(14,15,17)$. EphA3 expression was significantly higher in colorectal carcinoma than that in normal mucosal tissue and was associated with patient survival (17).

However, the relationship between EphA3 expression and survival in patients with HCC remains unknown. In the present study, we examined the expression of EphA3 by immunohistochemistry in HCC samples. Our results revealed that high levels of EphA3 expression in HCC tumor tissues were correlated with tumor size, tumor grade, metastasis, venous invasion and TNM stage, each of which is an indication of advanced 
tumor status. These results strongly suggest that EphA3 may play a key role in the progression of human HCC. Prognostic molecular biomarkers are invaluable for evaluating patient status and promoting tumor control. Kaplan-Meier analysis of the survival curves from patients in the present study showed a significantly worse overall survival rate for patients whose tumors had high EphA3 expression levels (log-rank test, $\mathrm{P}<0.001$ ), indicating that high levels of EphA3 protein may serve as a marker of poor prognosis for patients with HCC. Moreover, the multivariate analysis found EphA3 expression to be an indicator of worse patient outcome, independently of known clinical prognostic indicators such as TNM stage. These data suggest that high EphA3 expression is correlated with worse patient outcome and may serve as an independent prognostic factor for patients with HCC.

One significant finding of the present study was that metastasis and venous invasion were detected more frequently in EphA3-positive tumors compared to EphA3-negative cases. Invasion and metastasis are the processes by which tumors spread from the location of the primary tumor to distant locations in the body. These processes include a series of sequential steps, including tumor-induced angiogenesis, tumor invasion and establishment of metastatic foci at the secondary site involving various molecules $(35,36)$. One important molecule involved in tumor cell invasion and metastasis is VEGF. The expression of VEGF is commonly found to be upregulated in tumors and there is a trend toward an association between expression of VEGF and distant metastasis. Investigations by other laboratories have shown that VEGF promotes migration and invasion of tumor cells $(37,38)$. Angiogenesis plays an important role in tumors from the initial stage of carcinogenesis to the end stage of metastatic disease (39). The development of neovasculature in the tumor provides essential functions for growth, invasion and metastasis. VEGF is one of the isolated angiogenic peptides and is the most well-studied angiogenic factor to date. Moreover, VEGF is known to play a vital role in tumor-associated invasion $(40,41)$. In the present study, we showed that the invasive capabilities of EphA3 siRNA-transfected HCC cells were decreased and that the downregulation of EphA3 decreased the protein expression and proteolytic activity of VEGF. These results suggest that in HCC cells, the EphA3-VEGF axis may participate in tumor cell invasion. However, further study is necessary to elucidate the mechanism of the EphA3-VEGF interaction in HCC.

In summary, our findings strongly suggest that high levels of EphA3 expression significantly correlate with tumor progression and an unfavorable patient prognosis. In vitro, downregulation of EphA3 expression decreased the invasiveness of HCC cells via the regulation of VEGF. Therefore, EphA3 may be regarded as not only a novel candidate marker for prognosis but also a molecular target for HCC therapy. However, the underlying mechanisms responsible for these observations require further elucidation.

\section{References}

1. Blume-Jensen P and Hunter T: Oncogenic kinase signalling. Nature 411: 355-365, 2001.

2. Klein R: Excitatory Eph receptors and adhesive ephrin ligands. Curr Opin Cell Biol 13: 196-203, 2001.
3. Noren NK and Pasquale EB: Eph receptor-ephrin bidirectional signals that target Ras and Rho proteins. Cell Signal 16: 655-666, 2004.

4. Wilkinson DG: Eph receptors and ephrins: regulators of guidance and assembly. Int Rev Cytol 196: 177-244, 2000.

5. Cooke JE and Moens CB: Boundary formation in the hindbrain: Eph only it were simple. Trends Neurosci 25: 260-267, 2002.

6. Krull CE, Lansford R, Gale NW, et al: Interactions of Eph-related receptors and ligands confer rostrocaudal pattern to trunk neural crest migration. Curr Biol 7: 571-580, 1997.

7. Gale NW, Holland SJ, Valenzuela DM, et al: Eph receptors and ligands comprise two major specificity subclasses and are reciprocally compartmentalized during embryogenesis. Neuron 17: 9-19, 1996.

8. Davy A and Robbins SM: Ephrin-A5 modulates cell adhesion and morphology in an integrin-dependent manner. EMBO J 19: 5396-5405, 2000.

9. Lawrenson ID, Wimmer-Kleikamp SH, Lock P, et al: Ephrin-A5 induces rounding, blebbing and de-adhesion of EphA3expressing $293 \mathrm{~T}$ and melanoma cells by CrkII and Rho-mediated signalling. J Cell Sci 115: 1059-1072, 2002.

10. Wang J, Dong Y, Wang X, et al: Expression of EphA1 in gastric carcinomas is associated with metastasis and survival. Oncol Rep 24: 1577-1584, 2010.

11. Nakamura R, Kataoka H, Sato N, et al: EPHA2/EFNA1 expression in human gastric cancer. Cancer Sci 96: 42-47, 2005

12. Yuan W, Chen Z, Wu S, et al: Expression of EphA2 and E-cadherin in gastric cancer: correlated with tumor progression and lymphogenous metastasis. Pathol Oncol Res 15: 473-478, 2009.

13. Wang J, Li G, Ma H, et al: Differential expression of EphA7 receptor tyrosine kinase in gastric carcinoma. Hum Pathol 38: 1649-1656, 2007.

14. Hafner C, Schmitz G, Meyer S, et al: Differential gene expression of Eph receptors and ephrins in benign human tissues and cancers. Clin Chem 50: 490-499, 2004.

15. Wimmer-Kleikamp SH and Lackmann M: Eph-modulated cell morphology, adhesion and motility in carcinogenesis. IUBMB Life 57: 421-431, 2005.

16. Bae HJ, Song JH, Noh JH, et al: Low frequency mutation of the Ephrin receptor A3 gene in hepatocellular carcinoma. Neoplasma 56: 331-334, 2009.

17. $\mathrm{Xi} \mathrm{HQ}$ and Zhao P: Clinicopathological significance and prognostic value of EphA3 and CD133 expression in colorectal carcinoma. J Clin Pathol 64: 498-503, 2011.

18. Chu D, Li Y, Wang W, et al: High level of Notch1 protein is associated with poor overall survival in colorectal cancer. Ann Surg Oncol 17: 1337-1342, 2010.

19. Zhou L, Wang DS, Li QJ, Sun W, Zhang Y and Dou KF: Downregulation of the Notch signaling pathway inhibits hepatocellular carcinoma cell invasion by inactivation of matrix metalloproteinase- 2 and -9 and vascular endothelial growth factor. Oncol Rep 28: 874-882, 2012.

20. Raspollini MR, Amunni G, Villanucci A, Baroni G, Boddi V and Taddei GL: Prognostic significance of microvessel density and vascular endothelial growth factor expression in advanced ovarian serous carcinoma. Int J Gynecol Cancer 14: 815-823, 2004.

21. Dodelet VC and Pasquale EB: Eph receptors and ephrin ligands: embryogenesis to tumorigenesis. Oncogene 19: 5614-5619, 2000.

22. Clevers $\mathrm{H}$ and Batlle E: EphB/EphrinB receptors and Wnt signaling in colorectal cancer. Cancer Res 66: 2-5, 2006.

23. Nakamoto $M$ and Bergemann AD: Diverse roles for the Eph family of receptor tyrosine kinases in carcinogenesis. Microsc Res Tech 59: 58-67, 2002.

24. Brantley-Sieders D, Schmidt S, Parker M and Chen J: Eph receptor tyrosine kinases in tumor and tumor microenvironment. Curr Pharm Des 10: 3431-3442, 2004.

25. Héroult M, Schaffner F and Augustin HG: Eph receptor and ephrin ligand-mediated interactions during angiogenesis and tumor progression. Exp Cell Res 312: 642-650, 2006.

26. Xi HQ, Wu XS, Wei B and Chen L: Aberrant expression of EphA3 in gastric carcinoma: correlation with tumor angiogenesis and survival. J Gastroenterol 47: 785-794, 2012.

27. Davies H, Hunter C, Smith R, et al: Somatic mutations of the protein kinase gene family in human lung cancer. Cancer Res 65: 7591-7595, 2005

28. Bardelli A, Parsons DW, Silliman N, et al: Mutational analysis of the tyrosine kinome in colorectal cancers. Science 300: 949, 2003. 
29. Bonifaci N, Gorski B, Masojc B, et al: Exploring the link between germline and somatic genetic alterations in breast carcinogenesis. PLoS One 5: e14078, 2010.

30. Lee JS and Thorgeirsson SS: Comparative and integrative functional genomics of HCC. Oncogene 25: 3801-3809, 2006.

31. Boyd AW, Ward LD, Wicks IP, et al: Isolation and characterization of a novel receptor-type protein tyrosine kinase (hek) from a human pre-B cell line. J Biol Chem 267: 3262-3267, 1992.

32. Wicks IP, Wilkinson D, Salvaris E and Boyd AW: Molecular cloning of HEK, the gene encoding a receptor tyrosine kinase expressed by human lymphoid tumor cell lines. Proc Natl Acad Sci USA 89: 1611-1615, 1992.

33. Dottori M, Down M, Hüttmann A, Fitzpatrick DR and Boyd AW: Cloning and characterization of EphA3 (Hek) gene promoter: DNA methylation regulates expression in hematopoietic tumor cells. Blood 94: 2477-2486, 1999.

34. Fox BP, Tabone CJ and Kandpal RP: Potential clinical relevance of Eph receptors and ephrin ligands expressed in prostate carcinoma cell lines. Biochem Biophys Res Commun 342: 1263-1272, 2006.

35. Fidler IJ: The pathogenesis of cancer metastasis: the 'seed and soil' hypothesis revisited. Nat Rev Cancer 3: 453-458, 2003.
36. Weiss L: Metastasis of cancer: a conceptual history from antiquity to the 1990s. Cancer Metastasis Rev 19: I-XI, 193-383, 2000.

37. Wey JS, Fan F, Gray MJ, et al: Vascular endothelial growth factor receptor-1 promotes migration and invasion in pancreatic carcinoma cell lines. Cancer 104: 427-438, 2005.

38. Takahashi Y, Kitadai Y, Bucana CD, Cleary KR and Ellis LM: Expression of vascular endothelial growth factor and its receptor, KDR, correlates with vascularity, metastasis, and proliferation of human colon cancer. Cancer Res 55: 3964-3968, 1995.

39. Folkman $\mathrm{J}$ : What is the evidence that tumors are angiogenesis dependent? J Natl Cancer Inst 82: 4-6, 1990.

40. Joo YE, Sohn YH, Lee WS, et al: Expression of vascular endothelial growth factor and 553 in pancreatic carcinomas. Korean J Intern Med 17: 153-159, 2002.

41. Zeng H, Datta K, Neid M, Li J, Parangi S and Mukhopadhyay D: Requirement of different signaling pathways mediated by insulin-like growth factor-I receptor for proliferation, invasion, and VPF/VEGF expression in a pancreatic carcinoma cell line. Biochem Biophys Res Commun 302: 46-55, 2003. 